\title{
Secretion of Mutant Leucine-Specific Binding Proteins With Internal Deletions in Escherichia coli
}

\author{
Mark D. Adams and Dale L. Oxender \\ Department of Biological Chemistry, University of Michigan, Ann Arbor, Michigan 48109-0606
}

\begin{abstract}
The leucine-specific binding protein, encoded by the livK gene, is located in the periplasm of $E$. coli. The present study is an attempt to identify intragenic regions that determine the efficiency of its secretion into the periplasm. C-terminal deletions or fusions of the livK gene to trpA (encoding the $\alpha$ subunit of tryptophan synthetase) were secreted with little loss of efficiency [1]. A series of deletions was constructed at the unique Sphl site within livK, near the $5^{\prime}$ end of the region coding for the mature protein. Between 16 and 113 amino acids were deleted in the amino-terminal one-third of the protein. A few of these deletions were located within a few amino acids of the signal sequence processing site. Deletions extending within thirteen residues of the processing site were processed and secreted more slowly than normal. Secondary structure predictions suggested that the $\alpha$-helical core region of the signal sequence extends into the mature protein in the case of the slow processing mutants, perhaps interfering with the recognition site for leader peptidase or other secretory components. These results suggest that the conformation around the signal processing site may be a critical factor in determining the efficiency of secretion. During the course of this study, it was found that the difference in molecular weight between precursor and mature forms of some binding protein mutants, as judged by SDS-PAGE, was much greater than could be accounted for by processing of the signal sequence. This anomalous mobility on gels, however, could be eliminated by performing SDS-PAGE in the presence of $6 \mathrm{M}$ urea.
\end{abstract}

Key words: secretion, leucine binding protein, leader peptidase, signal sequence

The study of protein secretion has been driven by two complementary goals: 1) to understand the steps and factors that play a role in the transport of relatively large hydrophilic proteins across a membrane designed to prohibit even very small molecules from traversing, and 2 ) the application of the process of secretion for the production of heterologous proteins in $E$. coli. Current research is directed at the identification of the components of the export machinery involved in secretion and the characteristics of secreted proteins that allow them to interact productively with the export machinery. These characteristics are difficult to identify due to the complexity of the system, the degeneracy of the information in secreted proteins, and the multiple tasks that occur coincidentally (transcrip-

Received November 19, 1990; accepted April 1, 1991.

Mark D. Adams's present address is Section of Receptor Biochemistry and Molecular Biology, NINDS/NIH, Park Bldg., Bethesda, MD 20892.

Dale L. Oxender's present address is Dept. of Biotechnology, Warner-Lambert Co., 2800 Plymouth Rd., Ann Arbor, MI 48106. tion, translation, translocation, folding, and sorting).

The signal sequence is the defining characteristic of secreted proteins [2-4]. Although no consensus amino acid sequence has been discerned for the $\mathrm{N}$-terminal signal sequence, there is a strong conservation of several key structural features: 1) basic residues at the extreme amino terminus, 2) a hydrophobic core region that presumably folds into an $\alpha$-helical conformation, and 3) a small amino acid residue (usually alanine or glycine) that immediately precedes the site of processing by the signal peptidase [5-7]. The signal sequence is not, however, the sole segment of a protein to interact with the export machinery.

A variety of approaches have suggested that certain amino acid residues that follow the processing site mediate the effect of the signal sequence in the constructive interaction of the precursor protein with the export machinery. First, second-site mutations that restore secretion of signal sequence mutants are located within the mature portion of maltose-binding protein, MalE, suggesting an interaction be- 
tween the signal sequence and the mature protein [8-11]. Second, certain combinations of signal sequence and mature protein are not secreted well and can, in fact, be detrimental to the cell [15-18]. Third, Rasmussen and Silhavy have shown that the efficiency of secretion drops dramatically when internal LamB sequences are deleted to within twenty-eight residues of the signal processing site which also implicates an interaction of portions of the mature protein with a component of the export machinery [19]. Finally, Li et al. have demonstrated that the presence of a net positive charge in the first five residues of the mature protein can greatly reduce the rate of alkaline phosphatase secretion [20].

The majority of observations suggesting an interaction of the mature portion of the protein in the secretion process point to the aminoterminus of the mature protein, the amino acids within approximately fifty residues of the signal processing site $[1,13,19,11,14,21]$, although there is considerable evidence that other portions of the mature protein, closer to the carboxy terminus, may also be involved in release of the processed protein from the membrane [21-23]. One model of secretory protein interaction with the lipid bilayer, the helical hairpin model [24-27], suggests that the signal sequence cleavage site folds into a sharp hairpin conformation, allowing interaction between the signal sequence and the mature protein and facilitating interaction of the cleavage site with the signal peptidase. In addition, Inouye has examined the structural requirements for cleavage by signal peptidase, a critical step in release of a secreted protein from the bacterial inner membrane [28]. These results show that as the predicted conformation of the cleavage site becomes less like a $\beta$-turn, the rate of secretion is reduced $[28,29]$.

In this paper we present studies on a series of internal deletions within the leucine-specific binding protein (LS-BP), some of which are processed more slowly than others. The available evidence suggests that alteration of the secondary structure at the processing site can explain the slow processing behavior.

\section{METHODS \\ Construction of Deletion Mutants}

The livK gene encoded on the plasmid pOX7 $[30,31]$ was used as the starting point for construction of internal deletions. The SphI site in the vector sequence of pOX7 was removed by partial digestion with SphI followed by treatment with $\mathrm{S} 1$ nuclease to remove the singlestranded ends and ligation to yield pOX7S1. The plasmid pOX7S1 that exhibited a single SphI site within the livK gene was used for preparation of deletions. As diagrammed in Figure 1, pOX7S1 plasmid DNA was purified from low melting temperature agarose and digested with SphI and then with Bal31 exonuclease for a predetermined length of time to achieve removal of approximately 10-100 nucleotides in each direction. The plasmid was recircularized by ligation with T4 DNA ligase in the presence of BglII linkers. The resulting clones were screened first by restriction analysis and subsequently by immunoprecipitation for the production of full-length (in-frame) internal deletions. DNA fragments from clones which encoded inframe deletions were cloned into bacteriophage M13 and the sequence in the vicinity of the BglII linker of each clone was determined. Dideoxy sequencing reactions [32] were performed with Sequenase $^{\mathrm{TM}}$ from US Biochemicals and nucleotides from Pharmacia. Site-directed mutagenesis was performed by the method of Kunkel [33] as modified by Su and El-Gewely [34].

\section{Protein Secretion Assays}

The strains $\mathrm{DH} 5 \alpha \mathrm{F}^{\prime}$ (competent cells purchased from BRL, Inc.) and AE510 [35] were used for cloning and protein expression. Signal sequence processing was initially used as a marker for protein secretion by use of a pulsechase procedure. The pulse-chase experiments were carried out as follows. Briefly, exponentially growing cultures (1.5 ml) in MOPS rich medium [36] lacking methionine and leucine were labeled for 15 seconds with $50 \mu \mathrm{Ci}\left[{ }^{35} \mathrm{~S}\right] \mathrm{me}$ thionine (purchased from Amersham). An equal volume of pre-warmed media with $1 \mathrm{mM}$ unlabeled methionine was added and $1 \mathrm{ml}$ aliquots removed to $0.5 \mathrm{ml} 15 \%$ trichloroacetic acid (TCA) at 10,30 , and 60 seconds following initiation of the chase. TCA precipitable material was collected by centrifugation for five minutes at $4^{\circ} \mathrm{C}$. The pellet was washed once with cold acetone, suspended in 1\% SDS, $10 \mathrm{mM}$ Tris, $1 \mathrm{mM}$ EDTA, $\mathrm{pH} 8.0$, boiled, and immunoprecipitated with antibody to the LIV-BP [37]. Immunoprecipitates were subjected to SDS-polyacrylamide gel electrophoresis and the gel dried and autoradiographed.

Osmotic shock to release soluble proteins from the periplasm was performed according to Neu 


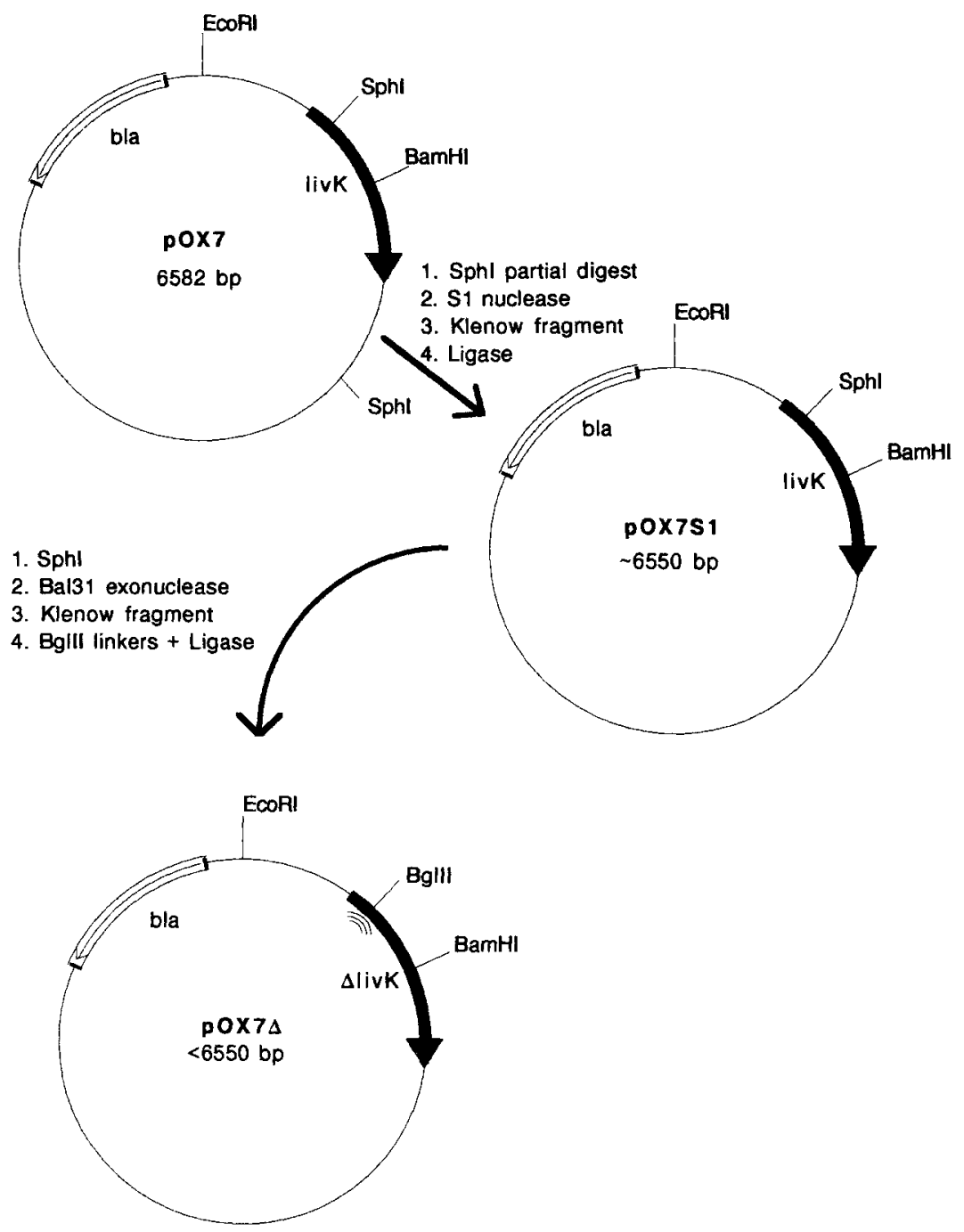

Fig. 1. Construction of internal deletions in livK. Internal deletions around the Sphl restriction site in the livK gene were constructed using Bal31 exonuclease. The ends were repaired using the Klenow fragment of DNA polymerase 1 and the plasmid was religated with BglII linkers to introduce a Bglil restriction enzyme cleavage site at the deletion junction.

and Heppel [38] and treatment with carbonylcyanide- $m$-chlorophenylhydrazone (CCCP) to block secretion by abolishing the membrane potential was carried out according to Landick et al. [39].

For in vitro expression, an S30 extract of the ribonuclease I mutant strain D10 [40] was prepared as described by Wilcox [41]. Plasmid DNA was purified for the in vitro coupled transcription/translation system by passage across a pZ253 column (from 5 Prime- $>3$ Prime Inc.). In vitro transcription/translation reactions were incubated at $37^{\circ} \mathrm{C}$ for five minutes before addition of plasmid DNA and then for 35 minutes following addition of $10 \mu \mathrm{g}$ of purified supercoiled plasmid DNA. Where indicated, incubation was continued for ten minutes in the presence of added signal peptidase. Purified leader peptidase (signal peptidase) was a gift of W. Wickner of UCLA.

\section{Computer Analysis}

Secondary structures of the deletion proteins were analyzed on a MicroVaxII using the University of Wisconsin Genetics Computer Group sequence analysis package [42].

\section{RESULTS}

Leucine-specific binding protein (LS-BP), encoded by the livK gene, is a 344 amino acid 
periplasmic protein that is initially synthesized with a 23 amino acid signal sequence. The protein participates in the transport of the branched-chain amino acid leucine, in cooperation with four membrane components of the LS system [35].

Previous results with the leucine-specific binding protein have shown that the protein can be secreted efficiently even when it is missing the carboxy terminus [43] or when it is fused to TrpA (tryptophan synthetase, $\alpha$-subunit) [1]. These experiments used gene fusions or deletions that began near the BamHI site in the livK gene (Fig. 1), corresponding to codon 256 of the mature protein. To examine the region closer to the signal processing site, a set of internal, inframe deletions was created at the SphI site.

Bal-31 exonuclease was used to create a series of bi-directional deletions around the SphI restriction site at codons $52-53$ of the livK gene. The resulting clones were screened with restriction enzymes to examine the position of the
BglII site relative to the codons for the signal processing site. Clones with a restriction pattern predicting that the deletion would not affect the signal sequence were screened for their ability to encode an immunoprecipitable inframe internal deletion. Fourteen clones that expressed in-frame deletion proteins were chosen for further study. Each clone was subjected to DNA sequencing in the area of the fusion junction to determine the reading frame and the exact residues that were missing. This information is summarized in Figure 2.

Signal sequence processing is an adequate, though not unequivocal measure of protein secretion. Since one activity of the signal peptidase appears to be to catalyze the release of protein from the inner membrane [27], the presence of the mature form should indicate that the protein has reached a late step in its secretion. A pulse-chase procedure was used to assay the processing (and thus, by inference, the secretion) of the internal deletion mutants. Figure 3

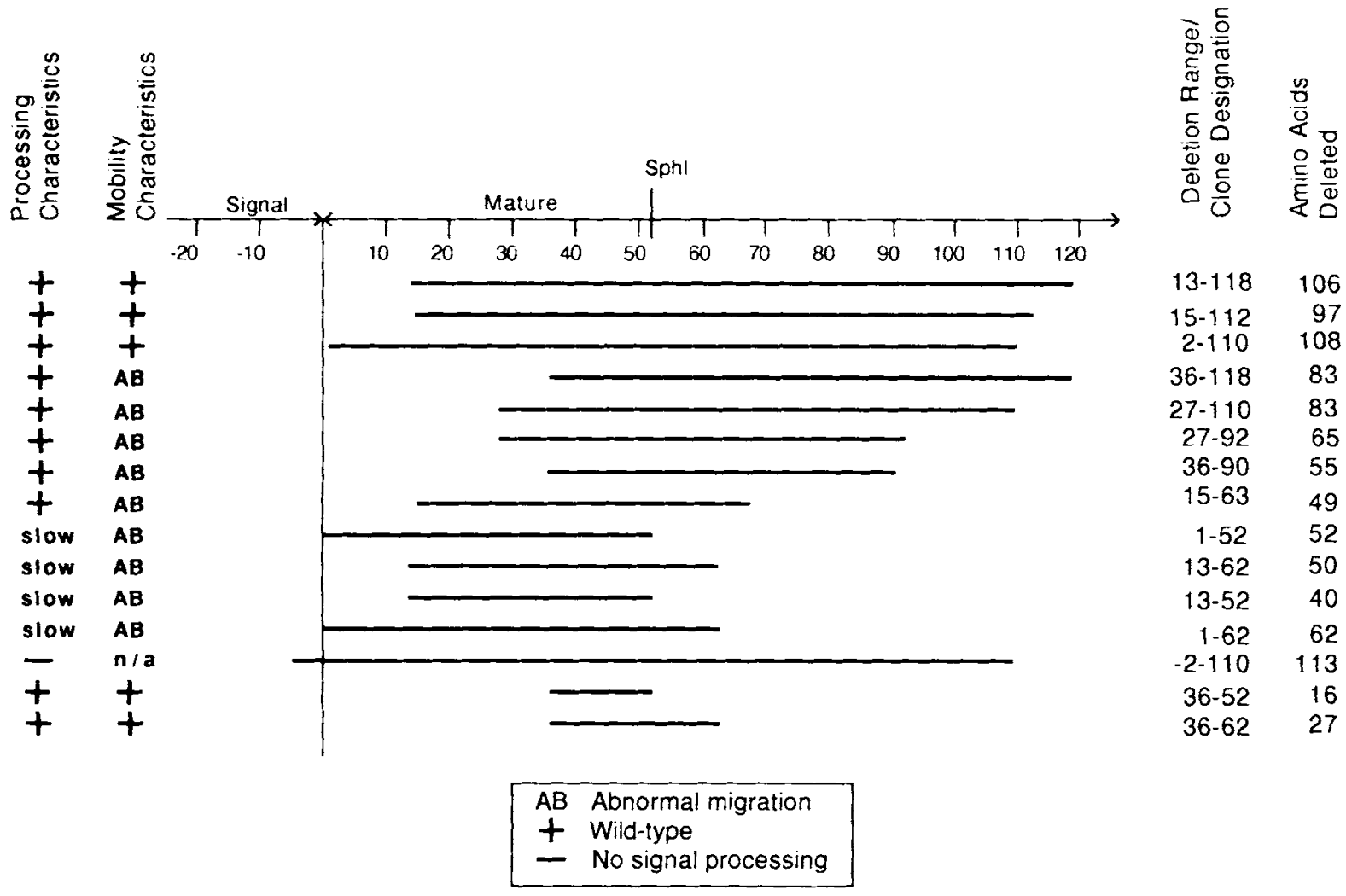

Fig. 2. Summary of deletion clone information. The extent of Bal31 deletion in each clone is diagrammed as a black bar. Clones are designated at the right by the range of residues which were deleted (as determined by nucleotide sequencing of each livK deletion fragment) and the length of the deletion. The left hand columns indicate signal sequence processing rate and gel mobility behavior of each deletion clone. $n / a$, not applicable. 


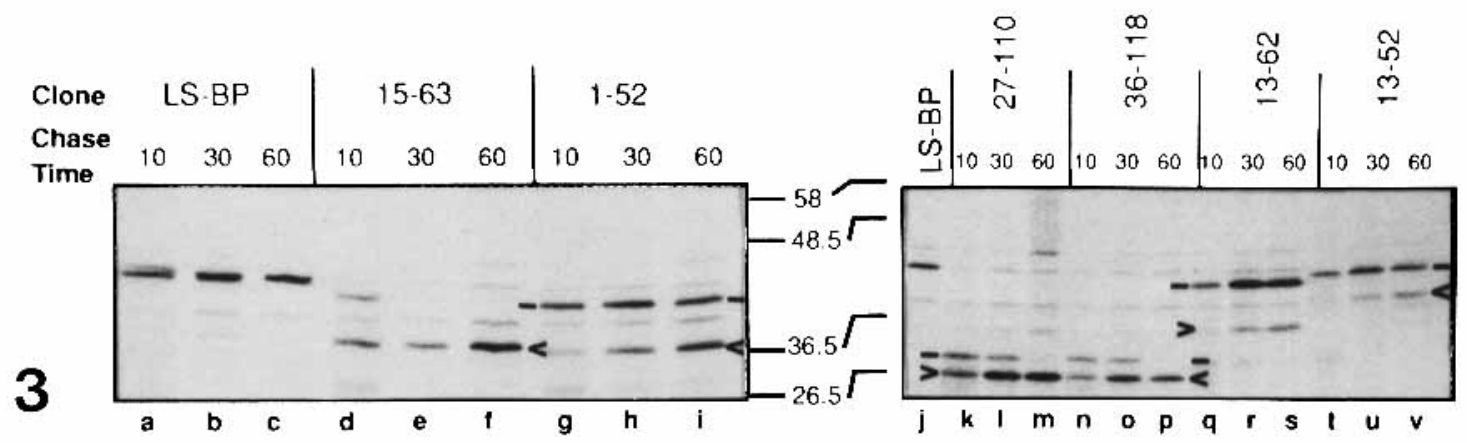

Fig. 3. Pulse-chase labeling of LS-BP and internal deletions. Cells carrying plasmids expressing the livK gene (LS-8P) or an internal deletion were grown to $\mathrm{OD}_{600}=0.8$ in MOPS rich medium lacking methionine and leucine. Cells were labeled with $50 \mu \mathrm{Ci}{ }^{35} \mathrm{~S}$ ]methionine for 10 seconds followed by a chase of media containing unlabeled methionine for 10,30 , and 60 seconds. Labeling reactions were stopped by transferring $1 \mathrm{ml}$ of labeled cells to $0.5 \mathrm{ml} 15 \%$ trichloroacetic acid (TCA) on ice. TCA precipitable material was collected by centrifugation, washed with cold acetone, and immunoprecipitated. Immunoprecipitated proteins were subjected to SDS-PAGE and the gel was dried and autoradiographed. Dashes indicate the position of the precursor form of each deletion protein and arrowheads indicate mature forms.

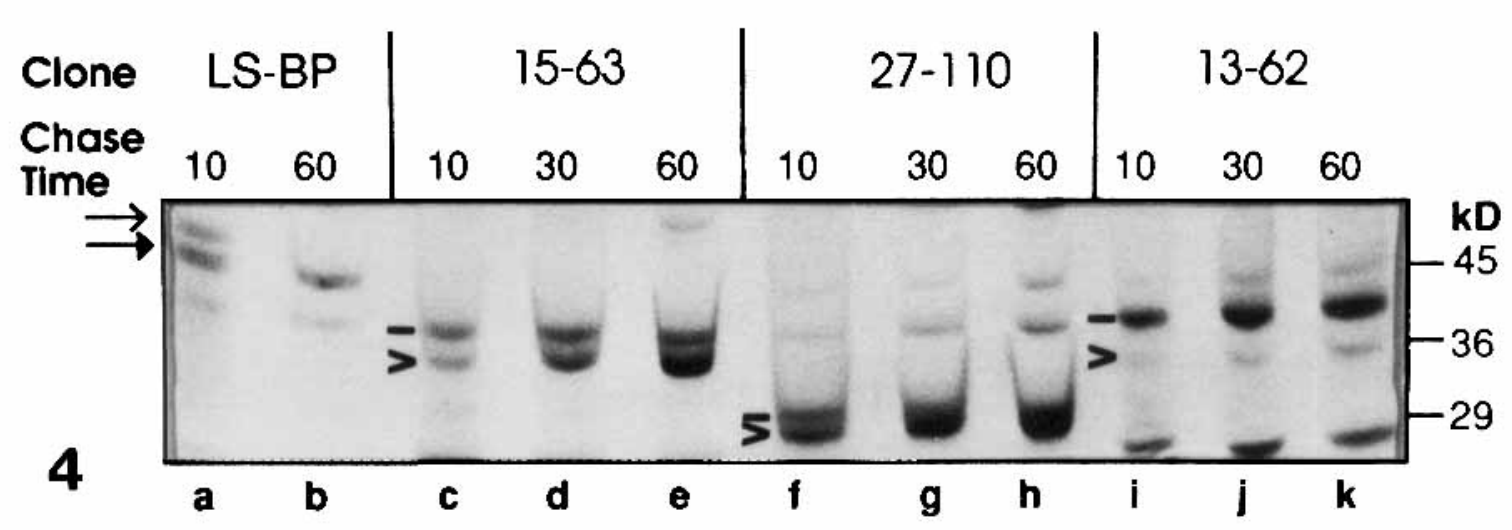

Fig. 4. SDS-urea gel of LS-BP and internal deletions. Pulse-chase labeling and immunoprecipitation were performed as described in the legend to Fig. 3 and in Methods. For electrophoresis, $6 \mathrm{M}$ urea was added to both the stacking and resolving gels. The open arrow and filled arrow on the left indicate the position of precursor and mature forms of LS-BP, respectively. Dashes indicate the position of the precursor form of each deletion protein and arrowheads indicate mature forms.

shows the results of the pulse-chase experiments for representative deletion clones.

The deletion mutants can be grouped two ways. First, some of the internal deletion mutants are processed much more slowly than wildtype LS-BP encoded on pOX7 ( $\Delta 1-52, \Delta 13-62$, $\Delta 13-52$ ). Slow processing is defined as less than fifty percent conversion of precursor to mature form at the thirty second chase time point, as determined by densitometric analysis of the autoradiograms. Second, some of the mutants have a much larger than expected apparent molecular weight difference $\left(\Delta \mathrm{M}_{\mathrm{r}}\right)$ between precursor and mature forms than LS-BP or other deletion mutants $(\Delta 15-63, \Delta 1-52, \Delta 13-62)$. Deletion proteins $\Delta 27-110$ and $\Delta 36-118$ are processed at nearly the wild-type rate and show no anomalous mobility. The anti-LS-BP antibody used for these immunoprecipitations cross-reacts with other proteins [43] which appear as bands in adjacent lanes with migrations similar to some of the deletion protein precursor or mature bands. The intensity of these bands is not changed, however, during the course of the chase while deletion precursor bands are reduced and mature bands are concomitantly increased.

The large $\Delta \mathrm{M}_{\mathrm{r}}$ between precursor and mature forms has several potential explanations. These include 1) processing at a site other than the normal signal processing site, 2) specific, nonsignal-peptidase mediated proteolysis, or 3) abnormal mobility in the gel system. The last 
explanation is the simplest and has been shown to be the case. When $6 \mathrm{M}$ urea was included in the SDS-PAGE resolving and stacking gels, the precursor and mature forms migrate as predicted from their molecular weight, by comparison to molecular weight markers (Fig. 4). A comparison of the predicted and apparent molecular weights of LS-BP and deletion mutants electrophoresed on urea and non-urea SDS gels showed that the precursor form is retarded in the gel so that its mobility predicts a molecular weight larger than the true molecular weight (data not shown). Anomalous mobility in SDS gels is more often observed for membrane proteins; however, it has also been seen for the histidine-binding protein, HisJ. The substitution of a single Cys residue for Arg in HisJ resulted in a 2,000 Dalton shift in mobility on SDS-PAGE [44].

The reduced rate of signal sequence processing was examined further in a cell-free coupled transcription/translation system (S30 extract) derived from the RNase $\mathrm{I}^{-}$strain D10. Two clones encoding proteins which differed by only four residues were chosen for analysis in the in vitro system:

\section{$12 \quad 13 \quad 14$ linker 64}

... Ser Gly Pro Asp Leu Lys . . . $\Delta 15-63$ (normal processing)

... Ser Gln Ile Cys Asn Lys . . $\Delta 13-62$ (slow processing)
12 linker
6364 .

When purified signal peptidase was added following the termination of the transcription/translation reaction, twice as much precursor $\Delta 15-63$ was converted to mature form during the 10 minute incubation when compared to precursor 13-62 (Fig. 5 analyzed by densitometer). Thus the relatively slow processing observed for $\Delta 13-62$ in pulse-chase experiments was reproduced in vitro indicating that it is the conformation of the precursor that is incompatible with signal sequence cleavage.

As can be seen in Figure 2, all of the slow processing mutants have deletions extending to within 13 residues of the signal processing site,

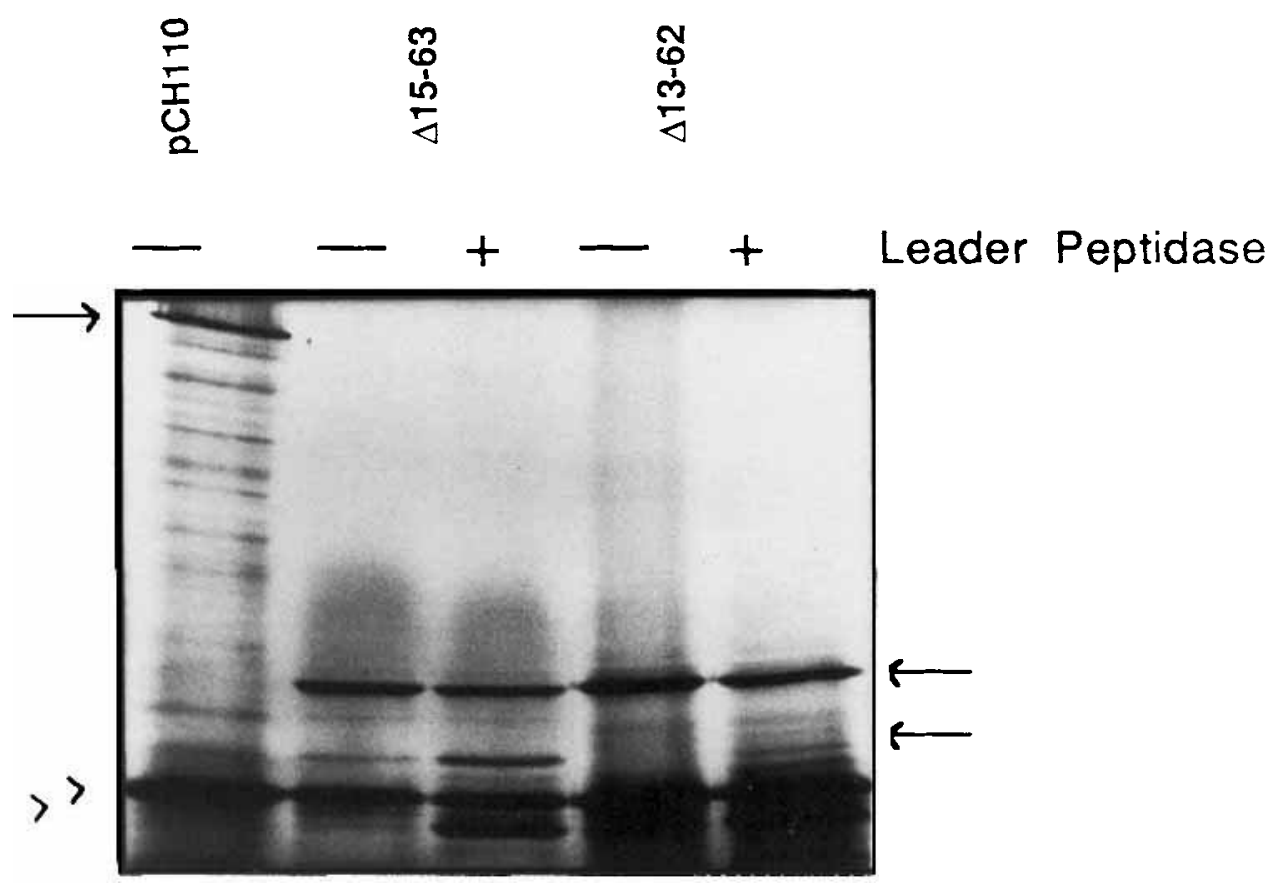

Fig. 5. In vitro processing of $\delta 13-62$ and $\delta 15-63$. Internal deletions $\delta 13-62$ and $\delta 15-63$ were transcribed and translated in vitro with an $\mathrm{S} 30$ extract of $E$. coli strain D10 as described in Methods. Following translation, purified $E$. coli leader peptidase (signal peptidase) was added for ten minutes where indicated. The arrow on the left indicates the position of $\beta$-galactosidase produced from the control plasmid $\mathrm{pCH} 110$. Arrowheads on the left indicated the position of precursor and mature $\beta$-lactamase, which is also translated from the internal deletion plasmids. Arrows on the right indicate the position of precursor (upper) and mature (lower) forms of both internal deletion proteins, 813-62 and $815-63$. 
suggesting that some structural aberration might interfere with processing by the signal peptidase. In an effort to identify potential changes in the physical characteristics of the region surrounding the processing site in the deletion mutants, several of the sequences were examined for their hydrophobicity, secondary structure predictions, and flexibility using the PEPTIDESTRUCTURE program of the University of Wisconsin Genetics Computer Group sequence analysis package. The charge distribution and amino acid composition in the vicinity of the deletion junction were also analyzed.

Many of the predictive algorithms showed essentially superimposable profiles for the two deletion mutants which differ by four residues, $\Delta 13-62$ and $\Delta 15-63$. Flexibility is slightly higher in the $\Delta 15-63$ mutant in the small region of the linker amino acids. The largest difference was observed in the Garnier-Osguthorpe-Robson (GOR) prediction of alpha helix and beta strand propensities [45]. These differences, illustrated in Figure 6, showed an increased potential for the $\alpha$-helical core region of the signal sequence to extend through the signal sequence process-

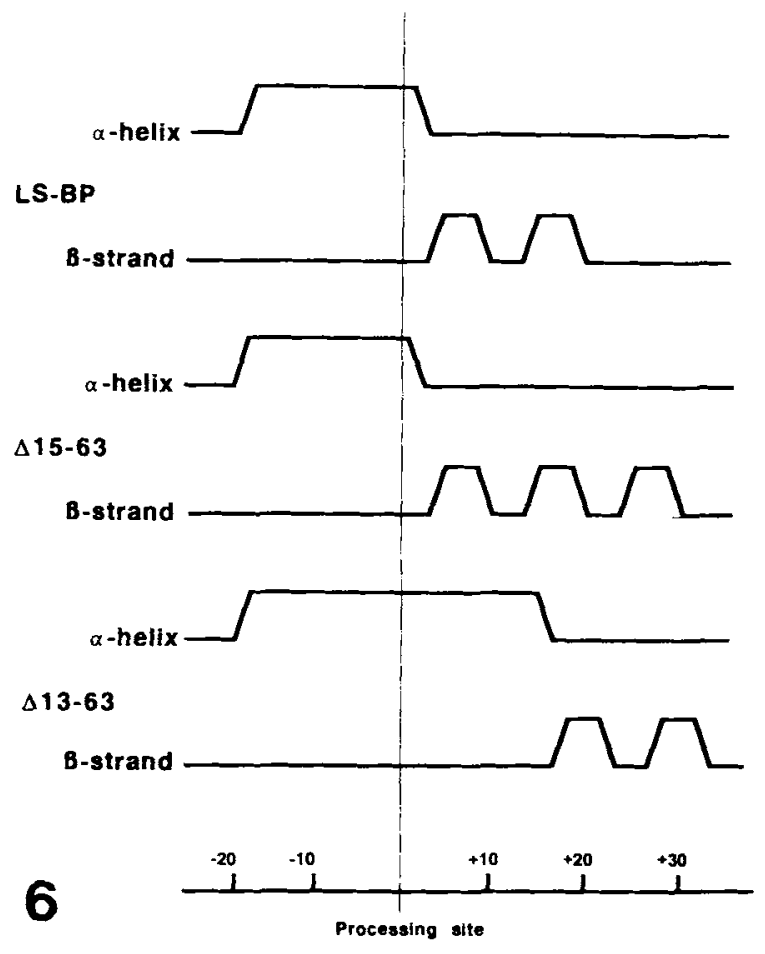

Fig. 6. Garnier-Osguthorpe-Robson secondary structure predictions. Secondary structure predictions were calculated by the algorithm of Garnier et al. [45] using the GCG package on a MicroVaxll computer. ing site into the mature protein for the $\Delta 13-62$ mutant relative to $\Delta 15-63$ and wild-type LS-BP. The Chou-Fasman predictions showed a decreased probability of a turn structure at residues 10 to 20 of the mature protein in $\Delta 13-62$. Overall, the GOR predictions are a better match than the Chou-Fasman predictions to the three dimensional structure of LS-BP as determined by single crystal X-ray diffraction analysis [46]. Analysis of the other deletion mutants showed that this altered pattern is present in each mutant that displays the slow processing behavior.

Examination of the difference in sequence between deletions $\Delta 13-62$ and $\Delta 15-63$ shows that glycine and proline are present at positions 13 and 14 in $\Delta 15-63$ and in wild-type LS-BP, but not in the slow processing mutant $\Delta 13-62$. Since both gly and pro are known to disrupt $\alpha$-helical structure, it seemed likely that these two residues were responsible for the slow processing rate of mutants which have deletions of these two residues. To test this hypothesis, two additional mutations were created by site directed mutagenesis of the wild-type livK gene encoding LS-BP. In one site-directed mutant, residues 13 and 14 were changed to alanine-alanine and in the other, the two residues were deleted. These two mutations were designated KAA13-14 and $\mathrm{K} \Delta 13-14$, respectively. GOR secondary structure predictions show the potential for increased $\alpha$-helical content at the processing site of both site-directed mutant proteins. An $E$. coli strain (strain AE510) that does not express either leucine binding protein [35] was used to assay the processing behavior of the two position 13-14 mutants. Pulse-chase labeling of strain AE510 [35] carrying plasmids expressing KAA13-14 and K $\Delta 13-14$ showed reduced rates of processing for both mutant proteins (Figure 7). By the ten

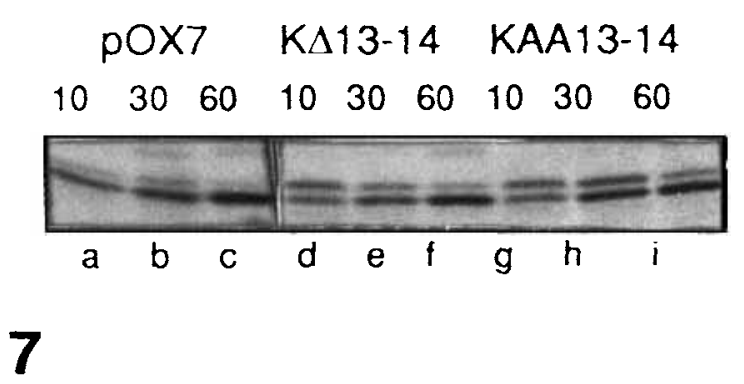

Fig. 7. Pulse-chase labeling of LS-BP site-directed mutant proteins. E. coli AE510 cells carrying livK, KAA13-14, or K\$13-14 plasmids were labeled and immunoprecipitated as described in the legend to Fig. 3. 
second chase time point, $74 \%$ of LS-BP has been converted to mature form, while $48 \%$ of KAA13-14 and 38\% of $\mathrm{K} \Delta 13-14$ have been converted (as determined by densitometric analysis of the autoradiograph). Unlike LS-BP, both sitedirected mutants show significant amounts of precursor remaining unprocessed at the sixty second chase time point.

In order to show unequivocally that processing of precursor to mature form does in fact constitute an effective assay for LS-BP secretion, we performed experiments to examine the subcellular localization of LS-BP and the internal deletion mutants. As was observed with C-terminal deletions and fusions to TrpA, internal deletion mutants were degraded rapidly in the periplasm, making detection of the secreted mature form more difficult by osmotic shock than by pulse-chase labeling. Cells were labeled with ${ }^{35}$ S $]$ methionine, treated with CCCP or a control buffer, and then fractionated by osmotic shock and immunoprecipitated. CCCP is a proton ionophore which has been shown to block the secretion process $[47,39]$. Figure 8 shows the autoradiograph from the resulting SDSpolyacrylamide gel.

In the presence of CCCP, the precursor form of a secreted protein accumulates and the protein is neither processed to the mature form nor released into the periplasm. Osmotic shock releases the contents of the periplasm so secreted proteins can be separated from the resulting spheroplasts by centrifugation. The results of these experiments showed that the osmotic shock fluid of cultures not treated with CCCP contain the mature form of secreted proteins (lanes c, $\mathrm{g}$, and $\mathrm{k}$ ); osmotic shock of the CCCP treated cultures did not release any proteins (lanes $d, h$, and 1 in Fig. 8). Likewise, in the presence of CCCP, the precursor form accumulated in the cell pellet fraction (lanes $b, f$, and $j$ ). In the absence of CCCP, the pellet fraction contained precursor and mature forms that had not yet been released from the membrane (lanes a, e, and i). In the absence of CCCP, the mature form of LS-BP and each deletion mutant was present in the osmotic-shock supernatant, indicating that they were actually secreted to the periplasm. Mature protein was also the primary species in the untreated pellet fraction due to incomplete spheroplasting and incomplete release of mature protein from the membrane.

\section{DISCUSSION}

The elements of structure of a secreted protein that make it competent to interact with the export machinery are gradually being elucidated. In general, it appears that a signal sequence alone is sufficient to lead the vast majority of proteins across the inner membrane of $E$. coli even though the efficiency may be low for some. The rate at which that transfer is accomplished varies greatly depending on the protein sequence downstream of the signal sequence. However, no sequence conservation has been detected among secreted proteins that would point to a shared specificity for a common sequence-specific site. This means that some struc-

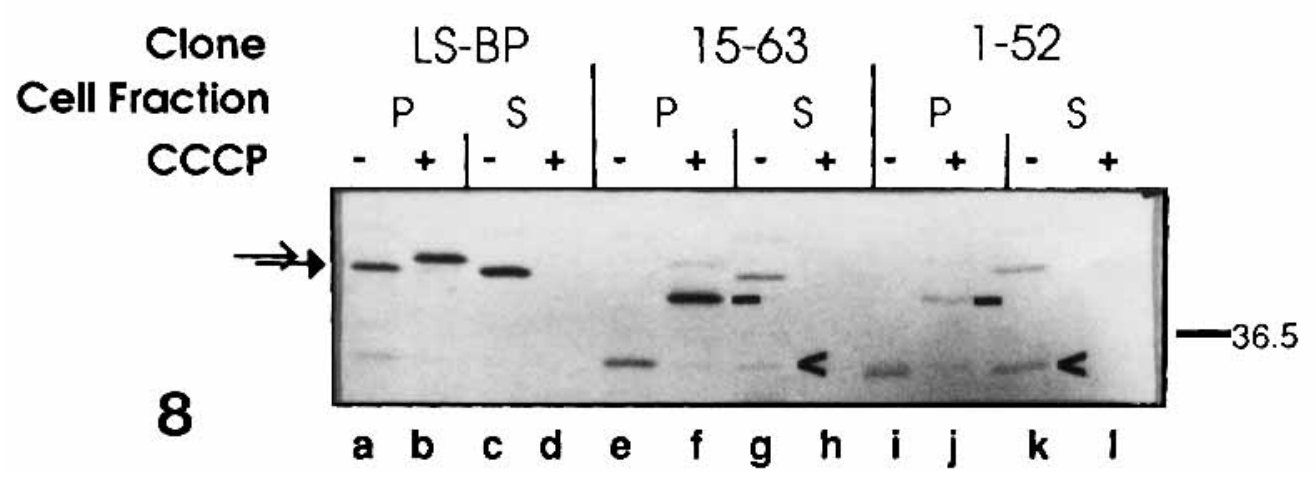

Fig. 8. Subcellular localization of LS-BP and internal deletions. Cells carrying plasmids expressing the livK gene (LS-BP) or an internal deletion were grown to $\mathrm{OD}_{600}=0.8$ in MOPS rich medium lacking methionine and leucine. Cells were labeled for five minutes with $50 \mu \mathrm{Ci}{ }^{35}$ S]methionine in the presence or absence of the proton ionophore CCCP, which blocks secretion. The cells were then separated into periplasmic and spheroplast fractions by the osmotic shock method of Neu and Heppel [38]. Each fraction was immunoprecipitated and subjected to SDS-PAGE. Osmotic shock pellet $(\mathrm{P})$ and supernatant $(\mathrm{S})$ are indicated in the Cell Fraction row. The open arrow and filled arrow on the left indicate the position of precursor and mature forms of LS-BP, respectively. Dashes indicate the position of the precursor form of each deletion protein and arrowheads indicate mature forms. 
tural motif, not readily discernible from the sequence, must carry the information for productive interaction with cellular components of the export machinery. This structural motif may perform any of a variety of functions. These could include direct interactions of the mature protein with export components or, in a less direct manner, interaction of the signal sequence with the mature protein. The conformation of the signal processing site could have a wide range of ramifications including control of the rate of folding of the precursor, recognition of a particular conformation of the precursor by the translocation apparatus, and specificity for the signal peptidase [12]. The requirement for a net neutral or negative charge in the first several residues of the mature protein may be an important component of the structural requirement [20].

Our results demonstrate that mutations in the mature protein can negatively affect the efficiency of secretion. One explanation for the reduced processing rate observed in these studies is that the secondary structure at the signal sequence processing site is perturbed in the slow processing mutants. Several of the deletion mutants analyzed exhibited greatly reduced rates of processing of the signal sequence as measured during pulse-chase assays (Fig. 3). All of these mutants were deleted to within 13 amino acid residues of the signal processing site (Fig. 2), and secondary structure predictions showed an increase in $\alpha$-helical structure in the vicinity of the processing site. By contrast, none of the mutants that were processed at a normal rate showed the increased helical content. Both $\Delta 2$ 110 and $\Delta 13-118$, with deletions close to the processing site, are processed at a nearly wildtype rate. These mutants contain the residues Gly-Pro at positions 16-17 and 19-20 of the mature protein, respectively, and do not show increased $\alpha$-helical propensity with the GOR algorithm.

The two candidate residues, $\mathrm{Gly}_{13}$ and $\mathrm{Pro}_{14}$, of the mature LS-BP were examined for their contribution to the rate of processing of wild-type LS-BP. Deletion of these two residues or their change to $\mathrm{Ala}_{13}-\mathrm{Ala}_{14}$ by site-directed mutagenesis reduced the rate of signal sequence processing, although not to the same extent observed in the deletion mutants. PLOTSTRUCTURE's GOR algorithm predicts increased $\alpha$-helical content at the processing site of both site-directed mutants. Mutation of residues 13 and 14 in the wild-type protein may not cause the same structural perturbation as the absence of these residues in the context of a larger deletion.

Rasmussen and Silhavy report a similar slowing of the processing rate in internal in-frame deletions of a LamB-LacZ fusion protein [19]. Analysis of these clones using the GOR algorithm as described above for the LS-BP failed to show the same sort of $\alpha$-helical elongation in the extreme amino terminus of the mature protein. Likewise, mutant maltose-binding proteins that are inefficiently secreted due to small deletions near the amino terminus did not show the altered $\alpha$-helical propensity [21]. The failure to detect alterations in secondary structure or other physical parameters at a distance of more than fifteen residues is not necessarily unexpected, since most of the algorithms employed use an averaging window of six to ten residues. The exception is the Garnier-Osguthorpe-Robson secondary structure prediction, which uses a window of eighteen residues. This extends the observed effects of amino acid substitutions; in our case, the extension includes the signal processing site. In addition, the GOR predictions of $\alpha$-helix and $\beta$-strand propensities match the true secondary structure of LS-BP as determined by $\mathrm{X}$-ray crystallography better than do the ChouFasman predictions. An alternative explanation is that a localized difference in secondary structure at the site of the deletion prevents that region of the protein from fulfilling its role in export. We do not believe this explanation can account for our results because there is no pattern of physical prediction into which fast processing and slow processing mutants can be grouped based on the structure at the position of the linker residues.

The S30 coupled transcription/translation system used for in vitro processing experiments would be expected to contain membranes, and it was expected that processing of the mature form of the protein would occur. However, processing was not observed to any great extent before addition of purified signal peptidase. Only the precursor form was visible on the gels. Possible reasons for the absence of processing include inactivation of the signal peptidase during preparation of the S30 extract and inability of the precursor protein to interact productively with the endogenous signal peptidase.

While our results indicate that signal peptidase activity on the slow processing mutants is reduced (Fig. 5), this does not necessarily mean 
that signal peptidase recognition is impaired. The conformation of the protein may be important at several steps of the secretion process; if signal sequence cleavage is a late event, then only a small fraction of the synthesized precursor may reach the stage at which it should be processed. Thus, addition of purified signal peptidase may have no effect on mutant precursor molecules that are blocked at an early stage of the secretion process, even though fully elongated precursor is present. If the signal sequence in the LS-BP deletions cannot perform its early functions effectively, then the protein may be sequestered in a folded or unfolded state inaccessible to signal peptidase, both in vitro and in vivo.

Several conserved structural features of the signal sequence permit constructive interaction of the precursor protein with components of the secretory pathway. The conformation and charge near the site of proteolytic cleavage are important contributors to the efficiency of this interaction $[12,20,28,29]$. Our results suggest that a decreased efficiency of secretion can be caused by a change in the conformation of the signal sequence processing site by amino acid substitutions in the mature protein at a distance of more than thirteen residues.

\section{ACKNOWLEDGMENTS}

This work was supported by National Institutes of Health grant GM11024. Thanks to R. Clark and L. Gunn who assisted in preparation of deletion mutant clones.

\section{REFERENCES}

1. Su T-Z, Copeland B, Landick R, Graddis TJ, Oxender DL: Acta Biochim et Biophys Sinica 20:364, 1988.

2. Milstein C, Brownlee GG, Harrison TM, Mathews MB: Nature 239:117, 1972.

3. Blobel G, Dobberstein B: J Cell Biol 67:835, 1975.

4. Blobel G, Dobberstein B: J Cell Biol 67:852, 1975.

5. von Heijne G: Eur J Biochem 133:17, 1983.

6. Watson MEE: Nucl Acids Res 12:5145, 1984.

7. Emr SD, Silhavy TJ: Proc Natl Acad Sci USA 80:4599, 1983.

8. Ryan JP, Duncan MC, Bankaitis VA, Bassford PJ Jr: J Biol Chem 261:3389, 1986.

9. Bankaitis VA, Bassford PJ Jr: Ann Inst Pasteur Microbiol 136B:3, 1985.

10. Bankaitis VA, Rasmussen BA, Bassford, PJ Jr: Cell $37: 243,1984$.
11. Cover WH, Ryan JP, Bassford PJ Jr, Walsh KA, Bollinger J, Randall, LL: J Bacteriol 169:1794, 1987.

12. Iino T, Sako T: J Biol Chem 263:19077, 1988.

13. Benson SA, Silhavy TJ: Cell 32:1325, 1983.

14. Benson SA, Bremer E, Silhavy, TJ: Proc Natl Acad Sci USA 81:3830, 1984.

15. Bankaitis VA, Bassford PJ Jr: J Biol Chem 259:12193, 1984.

16. Herrero E, Jackson M, Bassford PJ, Sinden D, Holland IB: J Bacteriol 152:133, 1982.

17. Kadonaga J'T, Gautier AE, Straus DR, Charles $A D$, Edge MD, Knowles JR: J Biol Chem 259:2149, 1984.

18. Andrews DW, Perara E, Lesser C, Lingappa VR: J Biol Chem 263:15791, 1988.

19. Rasmussen BA, Silhavy TJ: Genes \& Develop 1:185, 1987.

20. Li P, Beckwith J, Inouye H: Proc Natl Acad Sci USA 85:7685, 1988.

21. Duplay P, Hofnung M: J Bacteriol 170:4445, 1988.

22. Hengge R, Boos W: J Bacteriol 162:972, 1985.

23. Ito K, Beckwith JR: Cell 25:143, 1981.

24. von Heijne G, Blomberg C: Eur J Biochem 97:175, 1979.

25. Engelman DM, Steitz TA: Cell 23:411, 1981.

26. Shaw AS, Rottier PJM, Rose JK: Proc Natl Acad Sci USA 85:7592, 1988.

27. Dalbey RE, Wickner W: J Biol Chem 260:15925, 1985.

28. Inouye S, Duffaud G, Inouye M: J Biol Chem 261:10970, 1986.

29. Duffaud G, Inouye M: J Biol Chem 263:10224, 1988.

30. Oxender DL, Anderson JJ, Daniels CJ, Landick R, Gunsalus RP, Zurawski G, Yanofsky C: Proc Natl Acad Sci USA 77:2005, 1980.

31. Landick R, Oxender DL: J Biol Chem 26:8257, 1985.

32. Sanger F, Nicklen S, Coulson AR: Proc Natl Acad Sci USA 74:5463, 1977.

33. Kunkel TA: Proc Natl Acad Sci 82:488, 1985.

34. Su T-Z, El-Gewely MR: Gene 69:81, 1988.

35. Adams MD, Wagner LM, Graddis TJ, Landick R, Antonucci TK, Gibson AL, Oxender DL: J Biol Chem 265: $11436,1990$.

36. Neidhardt FC, Bloch PC, Smith DF: J Bacteriol 119: $736,1974$.

37. Oliver DB, Beckwith J: Cell 25:765, 1981.

38. Neu HC, Heppel LA: J Biol Chem 240:3685, 1965.

39. Landick RC, Daniels CJ, Oxender DL: Meth Enzymol 97:146, 1983.

40. Gesteland RF: J Mol Biol 16:67, 1966.

41. Wilcox G: Recomb DNA Tech 5:8, 1984.

42. Devereux J, Haeberli P, Smithies O: Nucl Acids Res 12:387, 1984.

43. Landick R, Duncan JR, Copeland BR, Nazos PM, Oxender, DL: J Cell Biochem 24:331, 1984.

44. Noel D, Nikaido K, Ames, GF-L: Biochemistry 18:4159, 1979.

45. Garnier J, Osguthorpe DJ, Robson B: J Mol Biol 120:97, 1978.

46. Sack JS, Trakhanov DS, Tsigannik IH, Quiocho, FA: J Mol Biol 206:193, 1989.

47. Daniels CJ, Bole DG, Quay SC, Oxender DL: Proc NatI Acad Sci 78:5396, 1981. 